\title{
Short communication: The effect of calcium supplementation at calving on neutrophil function
}

\author{
Cynthia L. Miltenburg, ${ }^{*}$ Todd F. Duffield, ${ }^{*}$ Dorothee Bienzle, $†$ Elizabeth L. Scholtz, ${ }^{*}$ and Stephen J. LeBlanc ${ }^{* 1}$ \\ *Department of Population Medicine, and \\ †Department of Pathobiology, University of Guelph, Guelph, Ontario, Canada N1G 2W1
}

\begin{abstract}
Low total blood calcium concentration after calving has been demonstrated to be a risk factor for reduced neutrophil function. The objective of this study was to evaluate whether administration of an injectable calcium supplement product soon after calving increased neutrophil oxidative burst or phagocytosis capacity. Cows $(\mathrm{n}=27)$ from 4 farms were blocked by parity and randomly assigned to receive either calcium gluconate (35\% wt/vol) in combination with calcium glucoheptonate ( $10 \%$ wt/vol; Theracalcium, Vétoquinol Canada Inc., Lavaltrie, Quebec, Canada) or a placebo within $12 \mathrm{~h}$ after calving and again $24 \mathrm{~h}$ later. Each dose of $120 \mathrm{~mL}$ was injected subcutaneously over 2 sites. Total serum calcium concentration, neutrophil oxidative burst, and neutrophil phagocytosis capacity were measured from coccygeal blood samples before (time 0 ) and $72 \mathrm{~h}$ after first treatment. There was no difference between treatment groups in lactation number, total calcium concentration, oxidative burst, or phagocytosis at time of enrollment. There was no effect of treatment on oxidative burst or phagocytosis by neutrophils. This preliminary study does not support an effect of supplemental calcium to improve neutrophil oxidative burst or phagocytosis capacity of low-parity parturient cows. Key words: calcium supplementation, immune function, transition cow
\end{abstract}

\section{Short Communication}

In the $72 \mathrm{~h}$ after calving, most dairy cows have reduced blood calcium concentrations. This transitory reduction is a result of a sudden substantial demand for calcium from the plasma pool at the onset of colostrum and milk production. Initiation of homeostatic mechanisms to restore circulating blood calcium to normal levels take several days, with the nadir of blood calcium

Received March 28, 2018.

Accepted June 28, 2018.

${ }^{1}$ Corresponding author: sleblanc@uoguelph.ca concentrations occurring 12 to $24 \mathrm{~h}$ following parturition (Goff, 2008).

Ionized calcium is important for messaging in cell metabolism and proliferation through changes in cytosolic ionized calcium concentrations. Intracellular calcium signaling is a key element in immune cell activation through influx of calcium from the extracellular space when antigen receptors are triggered (Vig and Kinet, 2009). In vitro, low extracellular ionized calcium was associated with decreased phagocytosis in bovine neutrophils (Ducusin et al., 2003). Kimura et al. (2006) demonstrated that mononuclear cells of periparturient cows have lower intracellular calcium stores, resulting in blunted calcium release in response to immune cell activation signals. Reduced calcium release in response to an immune cell activation signal likely contributes to periparturient immune suppression, but the reduced immune function that is experienced by almost all transition dairy cows with varying severity (Kehrli et al., 1989) is multifactorial and not well understood.

A decrease in circulating neutrophil oxidative burst activity postpartum has been found in cows that develop metritis (Hammon et al., 2006) and endometritis (Mateus et al., 2002; Hammon et al., 2006). Similar to other immune cells, an increase in intracellular calcium concentration is an early event in neutrophil activation (Burgos et al., 2011). Martinez et al. (2012) found neutrophil number, oxidative burst, and phagocytosis capacity to be reduced among cows with a blood calcium $<2.15 \mathrm{mmol} / \mathrm{L}$ between 1 and 3 DIM. These cows were also at greater risk of metritis. Supporting neutrophil function to reduce postpartum metabolic and reproductive disease is desirable, but it is unclear to what extent prevention or treatment of hypocalcemia may contribute. Prophylactic calcium supplementation, which is commonly administered to parturient dairy cows to reduce the incidence of clinical hypocalcemia, may be one way to achieve this. The objective of this study was to evaluate whether administration of an injectable calcium supplement product soon after calving increased neutrophil oxidative burst or phagocytosis capacity. 
A randomized controlled trial was conducted using parturient cows from 4 commercial dairy farms in Ontario, Canada, in June 2014. Herds were purposively selected based on proximity to the University of Guelph, willingness to comply with the calcium supplementation protocols, and herd size such that there would be cows available to enroll that had calved within the $12 \mathrm{~h}$ before the technician's visit. Producers agreed to refrain from using other forms of prophylactic calcium supplementation in enrolled cows and consented to a study protocol that had been reviewed and approved by the University of Guelph Animal Care Committee. All herds fed a TMR and prepartum cows did not receive anionic dietary supplements. The sample size for this proof-of-concept study was 13 cows per treatment based on detecting a $10 \%$ point difference $(\mathrm{SD}=9)$ in mean proportion of neutrophils performing oxidative burst.

Cows including first-parity animals that had calved in the previous $12 \mathrm{~h}$ were enrolled on the first day of the week for 3 consecutive weeks. Cows that showed signs of milk fever or injury related to calving or had already received a calcium supplementation product were excluded. Cows were randomly assigned to receive the calcium supplementation product or a placebo. Technicians administered and recorded experimental treatments according to randomized assignment sheets and were blinded to the treatment given. Cow ID, parity, calving ease, and time of calving were collected from farm personnel.

Prior to receiving treatment or the placebo, a single whole-blood sample was collected for calcium and neutrophil analysis. Whole blood was collected from the coccygeal vessels using a 20-gauge, 1 -inch hypodermic needle into sterile glass $10-\mathrm{mL}$ blood collection tubes without anticoagulant (BD Vacutainer Precision Glide; Becton Dickinson, Franklin Lakes, NJ). An additional 2 tubes were collected with the anticoagulant acid citrate dextrose (Vacutainer, Becton Dickinson).

Cows in the treated group received calcium gluconate (35\% wt/vol) in combination with calcium glucoheptonate $(10 \% \mathrm{wt} / \mathrm{vol})$ for a total of $9.46 \mathrm{~g}$ of calcium (Theracalcium, Vétoquinol Canada Inc., Lavaltrie, Quebec, Canada) given in 2 doses (within $12 \mathrm{~h}$ after calving at enrollment and again $24 \mathrm{~h}$ later). Each dose was $120 \mathrm{~mL}$ injected over 2 sites (60 mL per site) subcutaneously. Cows in the control group received a similar volume of placebo (medication vehicle solution with no active ingredient) at time of enrollment and $24 \mathrm{~h}$ later. At 3 DIM, the blood sampling procedure was repeated such that measurement of blood calcium and neutrophil analyses were completed twice per animal. Within $3 \mathrm{~h}$ of collection, serum was separated by centrifugation at $1,500 \times g$ for $15 \mathrm{~min}$. Serum was stored at $-20^{\circ} \mathrm{C}$ and analyzed at the Animal Health Laboratory, University of Guelph. Total calcium (tCa) concentration was measured using the Cobas Calcium Gen 2 kit (Roche Diagnostics, Indianapolis, IN). The analytical sensitivity of the calcium assay was $0.2 \mathrm{mmol} / \mathrm{L}$, and the interassay control coefficient of variation was $1.49 \%$.

Neutrophil isolation from whole blood with acid citrate dextrose was done within $3 \mathrm{~h}$ of collection. The procedure for neutrophil isolation and the oxidative burst and phagocytosis assays was as reported in Miltenburg et al. (2018). For the oxidative burst assay, $200 \mu \mathrm{L}$ of reconstituted neutrophils was incubated with fluorescent dye, and oxidative burst was stimulated with phorbol myristate acetate (PMA). Each sample had a negative control of neutrophils incubated without PMA. For the phagocytosis assay, $200 \mu \mathrm{L}$ of reconstituted neutrophils was incubated with $1 \times 10^{6}$ fluorescently labeled $1-\mu \mathrm{m}$ beads (TransFluo-Spheres Fluorescent Microspheres, Molecular Probes, Thermo Fisher Scientific, Waltham, MA) and $50 \mu \mathrm{L}$ of activated normal cow serum with Zymosan A (Sigma-Aldrich, St. Louis, MO). Each sample had a negative control of neutrophils incubated without fluorescent beads.

Neutrophil fluorescence was measured on a flow cytometer (FACScan, Becton Dickinson) with Cell Quest software (Becton Dickinson). A gate was placed around the neutrophil population on forward versus side scatter cytograms using FlowJo software (Tree Star, Ashland, OR). Per Miltenburg et al. (2018), the shift in the percentage of cells that underwent oxidative burst or phagocytosis was evaluated relative to each cow's negative control. A gate was placed around $\geq 97 \%$ of negative controls fluorescence (no PMA for the oxidative burst analysis and no fluorescent beads for the phagocytosis analysis), and the difference between the negative control and the positive observation for the percentage of cells outside the negative control gate was used to express success of oxidative burst or phagocytosis.

All statistical analyses were performed in SAS (version 9.4; SAS Institute, Cary, NC). Descriptive statistics were generated using the PROC MEANS and PROC FREQ procedures of SAS. The outcomes of interest were the difference between the samples and negative control for the mean percentage shift in cells that successfully performed oxidative burst and phagocytosis at 3 DIM. The oxidative burst variable required a natural logarithmic transformation to normalize the data with an adjustment factor of 0.5. Mixed linear regression models (MIXED procedure in SAS) were built for oxidative burst and phagocytosis with an autoregressive covariance structure. Farm, parity (primiparous or multiparous), and total calcium concentration at the time of enrollment as a continuous variable and a di- 

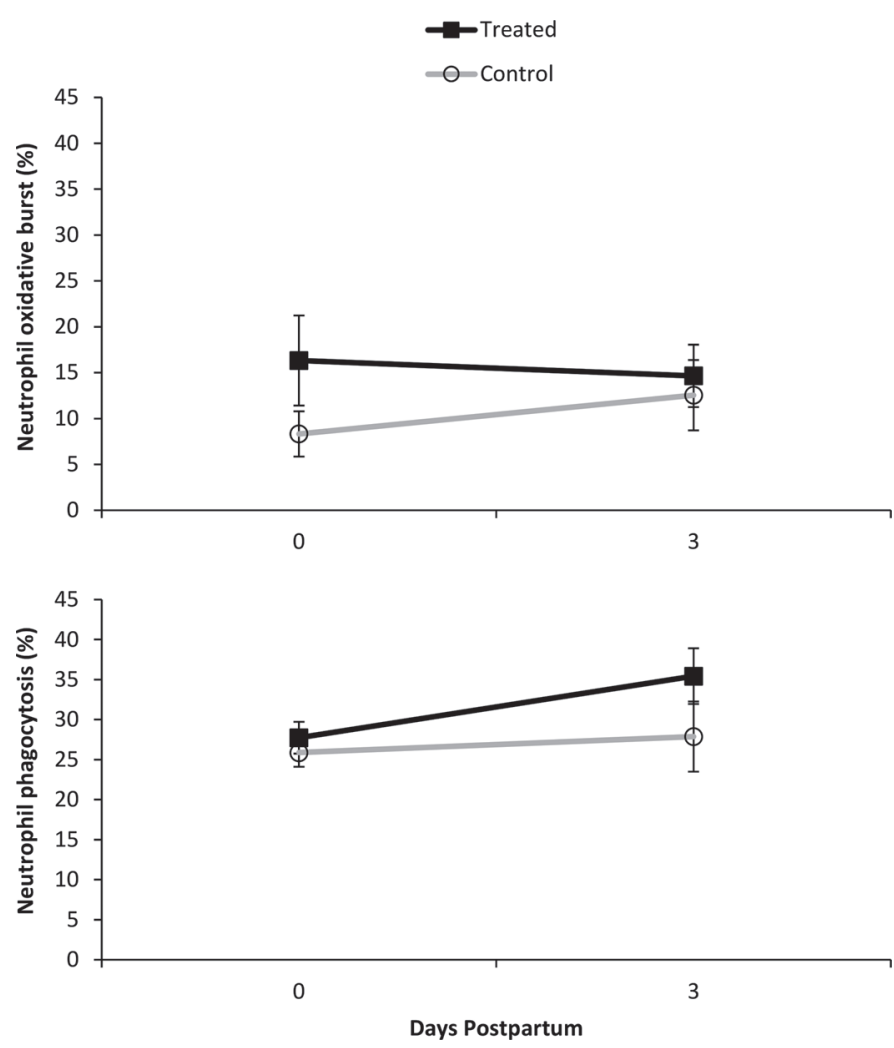

Figure 1. In vitro mean \pm SE oxidative burst and phagocytosis $(\%$ of neutrophils) in neutrophils of 27 cows treated with a subcutaneous calcium supplement or a placebo.

chotomous variable above and below the cut points of 2.0 and $2.15 \mathrm{mmol} / \mathrm{L}$ were offered as covariates to each model, and pretreatment oxidative burst or phagocytosis value at time 0 was controlled for as a covariate in each model. Variables remained in the final model if the $P$-value was $\leq 0.1$. Interactions of these covariates with treatment were tested and retained if significant $(P \leq 0.05)$.

A total of 29 animals were enrolled in the experiment. Two animals were unavailable for follow-up samples at 3 DIM due to death $(n=1)$ and culling $(n=1)$. There was no difference in lactation number, total calcium concentration, oxidative burst, or phagocytosis at the time of enrollment between treatment groups $(P>$ 0.2; Table 1). Of the 29 animals enrolled, 23 were first parity, 5 were second parity, and 1 was third parity. Enrollment from the 4 farms resulted in 11, 1, 3, and 14 cows from farms 1, 2, 3, and 4, respectively. The time between calving and first treatment as well as the interval between injections of the 2 doses of calcium did not differ between treatment groups $(P>0.65)$.

The prevalence of subclinical hypocalcemia at enrollment was $38 \%$ using a cut point of $2.15 \mathrm{mmol} / \mathrm{L}(\mathrm{n}=$ $11 ; 6 / 23$ primiparous and 5/6 multiparous). A cut point of $2.0 \mathrm{mmol} / \mathrm{L}$ characterized $24 \%$ of cows as subclinically hypocalcemic $(\mathrm{n}=7 ; 3 / 23$ primiparous and $4 / 6$ multiparous). At 3 DIM there was no difference in mean calcium concentration between groups $(P=0.62)$. The mean in vitro oxidative burst and phagocytosis (\% of neutrophils $) \pm$ standard error are displayed in Figure 1. There was no effect of treatment on oxidative burst $(P$ $=0.64$ ) accounting for oxidative burst at time 0 , and there was no effect of treatment on phagocytosis $(P=$ 0.19 ) accounting for phagocytosis at time 0 . There was no interaction $(P>0.30)$ of treatment with pretreatment blood calcium concentration (as a continuous variable or classified as $<2.0$ or $2.15 \mathrm{mmol} / \mathrm{L}$ ).

We hypothesized that treating cows with supplemental calcium would mitigate some of the expected reduction in neutrophil function. The results suggest that supplementation of parturient cows with subcutaneous

Table 1. Blood calcium concentrations and measures of neutrophil function on d 0 and 3 relative to calving in 27 dairy cows that received calcium supplementation or a placebo within $12 \mathrm{~h}$ of calving and $24 \mathrm{~h}$ later

\begin{tabular}{lrrrrr}
\hline & \multicolumn{2}{c}{ Treated } & & \multicolumn{2}{c}{ Control } \\
\cline { 2 - 3 } \cline { 5 - 6 } Item & Mean & SD & & Mean & SD \\
\hline Total serum Ca (mmol/L) & 2.11 & 0.25 & & 2.15 & 0.13 \\
d 0 & 2.20 & 0.22 & & 2.25 & 0.25 \\
d 3 & 16.32 & 20.83 & & 8.32 & 8.19 \\
Oxidative burst ${ }^{1}$ (\% of cells activated) & 14.64 & 14.03 & & 12.54 & 12.13 \\
d 0 & & & & & \\
d 3 & 27.74 & 8.44 & & 25.89 & 5.92 \\
Phagocytosis & (\% of cells activated) \\
d 0 & 35.44 & 14.34 & & 27.0 & 13.86 \\
d 3 & & & & & \\
\hline
\end{tabular}

${ }^{1}$ Oxidative burst was measured as the percentage of cells activated by phorbol myristate acetate, evaluated using flow cytometry.

${ }^{2}$ Phagocytosis was measured as the percentage of neutrophils that phagocytosed $\geq 1$ fluorescent bead, evaluated using flow cytometry. 
calcium as given here does not improve oxidative burst capacity or phagocytosis of the neutrophils.

The degree of hypocalcemia among the study sample was moderate, with $38 \%$ of animals having tCa $<2.15$ $\mathrm{mmol} / \mathrm{L}$ and $24 \%$ having tCa $<2.0 \mathrm{mmol} / \mathrm{L}$ immediately before treatment. In 657 cows from 7 herds sampled at a similar time in the same region, we found $59 \%$ of cows with tCa $<2.15 \mathrm{mmol} / \mathrm{L}$ (Miltenburg et al., 2016). Martinez et al. (2012) found that tCa $<2.15 \mathrm{mmol} / \mathrm{L}$ was associated with poorer neutrophil oxidative burst and phagocytosis capacity. Therefore, cows in our sample were at risk for improvement in neutrophil function if the calcium supplement was able to affect this. Although tCa was not characterized in the 12 to $24 \mathrm{~h}$ after treatment, a previous study demonstrated that the calcium supplement used in the current study significantly increased tCa in treated cows compared with a control group at $24 \mathrm{~h}$ after enrollment, and cows in the treatment group with a lower tCa at enrollment had a greater increase in tCa at $24 \mathrm{~h}$ (Miltenburg et al., 2016).

In the present study, overall oxidative burst capacity did not differ with treatment or between the 2 time points measured, but it varied widely among individual cows. The mean proportion of neutrophils showing oxidative burst was low on the day of calving (13\%); however, more than half the cows had lower oxidative burst at 3 DIM than at calving, consistent with the nadir of function occurring after calving. Mean phagocytosis capacity increased between the 2 time points measured, but two-thirds of the cows had declining phagocytosis capacity between the 2 time points. This is consistent with a decline in phagocytosis around $1 \mathrm{wk}$ postpartum (Kehrli et al., 1989; Kim et al., 2005; Sander et al., 2011).

Neutrophil life span is short, with cells lasting no more than a few days before being replaced (Kolaczkowska and Kubes, 2013). After production and differentiation in the bone marrow, it is unclear whether calcium concentrations can be altered in circulating neutrophils. When neutrophils were treated with the anticoagulant EDTA, an extracellular calcium ion chelator, neutrophil phagocytosis capacity was severely reduced (Ducusin et al., 2001). Previous work has suggested that cows with reduced calcium concentrations in blood are unable to replenish intracellular calcium and have less calcium in the endoplasmic reticulum available to affect cytosolic concentrations, thereby compromising neutrophil activation and the generation of reactive oxygen species (Ducusin et al., 2003; Martinez et al., 2012). These studies lead to the hypothesis that raising the total blood calcium concentration would alter this pathway. A greater understanding of the mechanisms by which neutrophils take up calcium in bone marrow or in circulation is needed to understand the potential to effect improvements in intracellular calcium and in neutrophil function. It is possible that the supplementation did not increase total blood calcium enough to effect change in intracellular calcium concentrations. It would be beneficial to measure the intracellular calcium concentration of neutrophils from supplemented cows versus controls to establish whether higher intracellular calcium was achieved.

A flux of intracellular calcium from the extracellular space to intracellular space is part of neutrophil activation; however, calcium-independent signaling pathways by means of protein kinases are also present (Sayeed, 2000). Cytosolic calcium is required to initiate phagocytosis (Sayeed, 2000) as well as the fusion of secondary granules with the phagosomal membrane (Jaconi et al., 1990). Furthermore, cytosolic calcium is required in the production of reactive oxygen species through activation of NADPH oxidation (Bréchard and Tschirhart, 2008). However, because neutrophil activation is not solely calcium dependent, a calcium supplement intervention might not be sufficient to affect neutrophil function when compromised peripartum immunity is multifactorial. Furthermore, if neutrophil oxidative burst function begins declining 2 to $3 \mathrm{wk}$ before calving, an intervention on the day of calving may be too late to effect change in neutrophil function. As the neutrophil life span is short and neutrophils circulating during this time are of lower oxidative burst capacity, increasing blood calcium concentration for 6 to $12 \mathrm{~h}$ through supplementation, even if this increases neutrophil intracellular calcium, might not be enough to effect meaningful improvement in immune defense and the overall health of the cow.

It is not clear whether different results would be achieved using a population of cows at greater risk of hypocalcemia, such as cows of greater parity. As risk of hypocalcemia increases with age or parity, an improvement in immune defense from calcium supplementation might be evident in older cows compared with the current sample, which by chance was skewed toward younger cows. Additionally, a larger sample that includes a greater number of cows at risk of reduced immune function, such as higher-producing cows and those at high risk of postpartum uterine disease, might yield different results. The main effect of calcium status at the time of enrollment and its interaction with treatment did not remain in the oxidative burst or phagocytosis models as an explanatory variable, suggesting that the effect of treatment was not different between subclinical and normocalcemic cows. Calcium status was explored using both the cut point of 2.0 and $2.15 \mathrm{mmol} / \mathrm{L}$ but without finding an effect. However, this should be explored more fully, potentially by 
enrolling and blocking cows by initial calcium status. The current research design did not allow characterization of the duration of hypocalcemia. It is possible that cows that were normocalcemic at enrollment became hypocalcemic at some later point. This information is relevant to understand the true incidence of subclinical hypocalcemia and the effect of calcium supplementation in normocalcemic versus hypocalcemic cows. Finally, interventions that produce a more sustained and earlier increase in blood calcium, such as feeding a prepartum diet with anionic salts to achieve a negative DCAD, would be worth investigation. A recent such study found some benefits for neutrophil function (Martinez et al., 2018). This preliminary study does not support an effect of supplemental calcium, as given to low-parity parturient cows, to improve oxidative burst or phagocytosis capacity of neutrophils.

\section{ACKNOWLEDGMENTS}

This study was funded by Vétoquinol Canada (Lavaltrie, Quebec, Canada) with student stipend support from the Ontario Ministry of Agriculture, Food and Rural Affairs (Guelph, Ontario, Canada). The authors thank the participating farms, technicians, and students.

\section{REFERENCES}

Bréchard, S., and E. J. Tschirhart. 2008. Regulation of superoxide production in neutrophils: Role of calcium influx. J. Leukoc. Biol. 84:1223-1237.

Burgos, R. A., I. Conejeros, M. A. Hidalgo, D. Werling, and C. Hermosilla. 2011. Calcium influx, a new potential therapeutic target in the control of neutrophil-dependent inflammatory diseases in bovines. Vet. Immunol. Immunopathol. 143:1-10.

Ducusin, R. J., T. Sarashina, Y. Uzuka, S. Tanabe, and M. Ohtani. 2001. Phagocytic response of bovine polymorphonuclear leukocytes to different incubation conditions and following exposure to some effectors of phagocytosis and different anticoagulants in vitro. Can. J. Vet. Res. 65:38-44.

Ducusin, R. J. T., Y. Uzuka, E. Satoh, M. Otani, M. Nishimura, S. Tanabe, and T. Sarashina. 2003. Effects of extracellular $\mathrm{Ca}^{2+}$ on phagocytosis and intracellular $\mathrm{Ca}^{2+}$ concentrations in polymor- phonuclear leukocytes of postpartum dairy cows. Res. Vet. Sci. $75: 27-32$.

Goff, J. P. 2008. The monitoring, prevention, and treatment of milk fever and subclinical hypocalcemia in dairy cows. Vet. J. 176:50-57.

Hammon, D. S., I. M. Evjen, T. R. Dhiman, J. P. Goff, and J. L. Walters. 2006. Neutrophil function and energy status in Holstein cows with uterine health disorders. Vet. Immunol. Immunopathol. 113:21-29.

Jaconi, M. E., D. P. Lew, J. L. Carpentier, K. E. Magnusson, M. Sjogren, and O. Stendahl. 1990. Cytosolic free calcium elevation mediates the phagosome-lysosome fusion during phagocytosis in human neutrophils. J. Cell Biol. 110:1555-1564.

Kehrli, M. E. Jr., B. J. Nonnecke, and J. A. Roth. 1989. Alterations in bovine lymphocyte function during the periparturient period. Am. J. Vet. Res. 50:215-220.

Kim, I. H., K. J. Na, and M. P. Yang. 2005. Immune responses during the peripartum period in dairy cows with postpartum endometritis. J. Reprod. Dev. 51:757-764.

Kimura, K., T. A. Reinhardt, and J. P. Goff. 2006. Parturition and hypocalcemia blunts calcium signals in immune cells of dairy cattle. J. Dairy Sci. 89:2588-2595.

Kolaczkowska, E., and P. Kubes. 2013. Neutrophil recruitment and function in health and inflammation. Nat. Rev. Immunol. 13:159 175

Martinez, N., C. A. Risco, F. S. Lima, R. S. Bisinotto, L. F. Greco, E. S. Ribeiro, F. Maunsell, K. Galvao, and J. E. Santos. 2012. Evaluation of peripartal calcium status, energetic profile, and neutrophil function in dairy cows at low or high risk of developing uterine disease. J. Dairy Sci. 95:7158-7172.

Martinez, N., R. M. Rodney, E. Block, L. L. Hernandez, C. D. Nelson, I. J. Lean, and J. E. Santos. 2018. Effects of prepartum dietary cation-anion difference and source of vitamin D in dairy cows: Health and reproductive responses. J. Dairy Sci. 101:2563-2578.

Mateus, L., L. Lopes da Costa, H. Carvalho, P. Serra, and J. Robalo Silva. 2002. Blood and intrauterine leukocyte profile and function in dairy cows that spontaneously recovered from postpartum endometritis. Reprod. Domest. Anim. 37:176-180.

Miltenburg, C. L., T. F. Duffield, D. Bienzle, E. Scholtz, and S. J. LeBlanc. 2016. Randomized clinical trial of a calcium supplement for improvement of health in dairy cows in early lactation. J. Dairy Sci. 99:6550-6562.

Miltenburg, C. L., T. F. Duffield, D. Bienzle, E. Scholtz, and S. J. LeBlanc. 2018. The effect of prepartum feeding and lying space on metabolic health and immune function. J. Dairy Sci. 101:52945306 .

Sander, A. K., M. Piechotta, G. Schlamberger, H. Bollwein, M. Kaske, A. Sipka, and H. J. Schuberth. 2011. Ex vivo phagocytic overall performance of neutrophilic granulocytes and the relation to plasma insulin-like growth factor-I concentrations in dairy cows during the transition period. J. Dairy Sci. 94:1762-1771.

Sayeed, M. M. 2000. Exuberant $\mathrm{Ca}(2+)$ signaling in neutrophils: A cause for concern. News Physiol. Sci. 15:130-136.

Vig, M., and J. P. Kinet. 2009. Calcium signaling in immune cells. Nat. Immunol. 10:21-27. 